\title{
IMPACTO SOCIAL DE LA MODERNIZACIÓN DE LOS SISTEMAS VIALES Y PRODUCTIVOS EN LA REGIÓN CHOROTEGA DE COSTA RICA, 1950-2013
}

\section{SOCIAL IMPACT OF MODERNIZATION OF ROADS AND PRODUCTION SYSTEMS IN THE CHOROTEGA REGION OF COSTA RICA, 1950-2013}

\section{Edgar Blanco Obando*}

\section{RESUMEN}

Durante la segunda mitad del siglo xx, el Estado resolvió una gran parte de los problemas de comunicación entre la región Chorotega y el resto del país a través de la construcción de modernas vías terrestres y la instauración de dinámicos sistemas productivos. Sin embargo, esta modernización y cambio tecnológico no logró revertir los problemas regionales de pobreza, desempleo y desigualdad.

PALABRAS CLAVE: CAMBIO TECNOLÓGICO * BIENESTAR SOCIAL * HISTORIA REGIONAL * DESARROLLO $*$ SISTEMAS PRODUCTIVOS

\section{ABSTRACT}

During the second half of the twentieth century, the state settled the Chorotega region's isolation from the rest of the country through the construction of modern land routes and the establishment of dynamic production systems. However, this process of modernization and technological change failed to reverse the serious social problems such as poverty, unemployment and inequality.

KEYWORDS: TECHNOLOGICAL CHANGE * SOCIAL WELFARE * REGIONAL HISTORY * DEVELOPMENT * PRODUCTION SYSTEMS 


\section{INTRODUCCIÓN}

Durante las primeras décadas de la segunda mitad del siglo xIx, en el actuar del Estado costarricense es posible apreciar una fuerte influencia de ideales liberales impulsados por las élites políticas y económicas radicadas en el Valle Central, reflejada en el fomento del desarrollo y el progreso a través de asegurar el dominio del Estado en todo el territorio nacional, de modo que prevaleciera un solo sistema económico e ideológico de Estado-Nación, capaz de articular a las regiones con el centro político-económico mediante la promoción del poblamiento en las zonas alejadas, la construcción de infraestructura productiva $y$ de comunicación, el fomento de la educación y el comercio, junto al impulso de actividades económicas modernas y dinámicas que asegurasen la participación del país en los mercados internacionales (Viales, 2000a y 2000b).

Para las primeras décadas del siglo xx, el país presentaba problemas importantes de comunicación entre las regiones y la capital — principal polo económico y político-, por lo que los gobiernos actuaron con el fin de construir una moderna infraestructura productiva en las regiones y eficientes vías de comunicación. Se logra así la incorporación de todo el territorio dentro del modelo económico $y$ político regido desde la capital y se asegura la salida de los productos del centro del país hacia las regiones como al extranjero, principalmente mediante la exportación a través de los puertos en el Caribe y en el Pacífico.

Hasta la década de 1970, esta situación se dio de forma importante en la región Chorotega, la cual solo contaba con el cabotaje en vías fluviales como la principal vía de acceso con el resto del país, como consecuencia sufría serios problemas de comunicación y de rezago de su sistema comercial. Por lo tanto, el Estado realizó importantes esfuerzos para revertir esta condición, mediante la construcción de modernas vías de comunicación, así como, el fomento de dinámicas $y$ actividades productivas orientadas a la inserción de la economía regional en los mercados tanto interno como internacional.

De esta manera, en la década de 1960, se logró la entrada en funcionamiento de la carretera Interamericana, que aseguró una vía terrestre $y$ transitable, tanto en invierno como en verano, entre la región Chorotega y el resto del país, desplazando a las modestas rutas fluviales de cabotaje como eje central del sistema vial en la región.

La modernización de las vías de comunicación facilitó la instauración de los diferentes modelos productivos propios de las tendencias económicas impulsadas por el Estado, por ejemplo, un intensivo sistema agroproductivo basado en la ganadería extensiva y el cultivo de alimentos a través de la instauración de nuevas y modernas tecnologías, así como el desarrollo de las actividades terciarias a partir del decenio de 1990, principalmente de tipo turístico orientado a los mercados internacionales.

Este cambio tecnológico en los sistemas viales y productivos, impulsó el desarrollo a través de la instauración de un modelo productivo capaz de construir bienes, servicios y transacciones de mercado, con el fin de crear riqueza, resolver las necesidades de las personas y generar bienestar social mediante la reducción de los problemas de pobreza y desempleo.

De este modo, el Estado costarricense ha actuado como agente de cambio en la región Chorotega, como una parte dentro de una cadena de actores que incorporó a las élites regionales, empresas, artefactos y la población local, con el predominio de relaciones tecno-económicas y socio-políticas determinadas (Lepratte, Blanc, Pietroboni y Hegglin, 2015).

Esta situación ha producido diversos efectos a nivel social en la región Chorotega, lo cual se evidencia através del análisis de los principales indicadores de bienestar de la población a partir de la premisa que las acciones estatales para el desarrollo pretenden la mejora de los niveles de vida de las comunidades (Worster, 2006). En este escenario, la tecnología ha desempeñado la función de elemento para la transformación de parámetros culturales, económicos y políticos, permitiendo el fomento del progreso y del cambio social (Tabares y Correa, 2014).

Entre las décadas de 1950 y 1980, se aplicó en el país un modelo de desarrollo sustentado en las teorías de la modernización y el 
desarrollismo, que promovió el cambio tecnológico en las unidades productivas con el fin de modernizarlas e industrializarlas, con el fin de incrementar la producción y la generación de riqueza, basado en un concepto del desarrollo meramente economicista (Delgado, 2004). De esta manera, el Estado impulsó la industrialización de la producción local y la mejora de su competitividad mediante la transmisión de tecnologías, bienes de capital, subsidios y protección arancelaria contra las importaciones.

Si bien, con estas acciones se incrementó la producción y creció la industria, el cambio tecnológico y el sistema proteccionista resultaron sumamente caros $y$ beneficiaron especialmente a los sectores más poderosos, por lo que no se logró superar los problemas de pobreza y rezago padecidos por la mayoría de la población y unidades productivas. Más bien, se afectó severamente la estabilidad de la hacienda pública y se comprometió la continuidad del sistema productivo, lo que obligó a reformular la estrategia de desarrollo y la producción en general, mediante la propuesta de instaurar un nuevo sistema productivo basado en unidades más eficientes y capaces de competir exitosamente en los mercados internacionales (Rovira, 1987).

El desbalance ocasionado por el elevado costo del sistema proteccionista llegó a ser insostenible al inicio de la década de 1980, cuando los ingresos provenientes del comercio dentro del Mercado Común Centroamericano — donde el país dirigía buena parte de sus exportaciones - sufrieron una baja significativa a causa de los conflictos bélicos que golpearon a la región en estos años (León et ál., 2014).

De igual manera, el país se vio afectado por el aumento desproporcionado en los precios mundiales de los combustibles y en las tasas internacionales de interés dentro de la crisis mundial a inicios de la década, por lo que perdió gran parte de su capacidad para generar recursos, ocasionando la reducción del Producto Interno Bruto (PIB), que solo entre 1982 y 1983 se contrajo en cerca del 10\% (León, 2012).

Estas situaciones provocaron una elevada inestabilidad económica y el estancamiento del sistema productivo, lo que obligó al país a establecer nuevas negociaciones con el Banco
Mundial (вм) y el Fondo Monetario Internacional (FMI), con el fin de obtener nuevos recursos externos para reactivar la economía y la producción para salir de la crisis. En estas negociaciones, el acceso a los créditos se condicionó a la reformulación de la política económica y productiva de acuerdo a dogmas económicos basados en una nueva interpretación de las clásicas teorías del libre mercado y del rol del Estado en el fomento del desarrollo, lo cual contó con el apoyo de nuevos y ascendentes grupos económicos nacionales (León, 2012).

El país inició la aplicación de toda una serie de políticas fiscales, institucionales, económicas y productivas, dirigidas a la apertura de los mercados, la internacionalización de la economía, la eliminación del intervencionismo estatal, la reducción del gasto público, el fomento de las exportaciones, la atracción de inversión privada y el apoyo a las actividades productivas más rentables y competitivas (Rovira, 1987).

De este modo, a mediados del decenio de 1990, se consolidó la transformación del aparato productivo de acuerdo a los postulados del neoliberalismo - tendencia económica predominante a nivel mundial - basados en el fomento de la empresa privada, la reducción del gasto público y el apoyo a las producciones eficientes $y$ transnacionalizadas. Por otro lado, se retiró el apoyo a las unidades agrícolas tradicionales, dedicadas al abastecimiento del mercado interno y se impulsó el crecimiento de la agricultura de exportación y el sector de los servicios, principalmente el turismo, al definirse como actividades competitivas y capaces de generar divisas mediante su competencia exitosa en los mercados mundiales más lucrativos.

El neoliberalismo ocasionó a nivel productivo la asignación de las ayudas y estímulos estatales a los grandes exportadores e inversores, capaces de competir exitosamente en los mercados internacionales y crear divisas; esto desestimuló la producción de los productos tradicionales como los granos básicos y alimentos en general, junto a la promoción de las exportaciones de los productos no tradicionales: frutas, flores y otros bienes exóticos de gran demanda en el mercado internacional; al 
igual que al sector de los servicios, principalmente el turismo y los servicios financieros, debido a su rentabilidad y capacidad para explotar las ventajas comparativas del país (León et ál., 2014).

Por esta razón, la definición del período 1950-2013, permite analizar las diferentes coyunturas y momentos históricos en que se dio la transformación y modernización de modelos económicos y sistemas viales junto a sus efectos sociales derivados, los cuales debido a su importancia e impacto, prácticamente revolucionaron las relaciones entre la capital y la zona de estudio.

La región Chorotega comprende el territorio de la provincia del Guanacaste, sus 11 cantones oficiales (Liberia, Nicoya, Santa Cruz, Bagaces, Carrillo, Cañas, Abangares, Tilarán, Nandayure, La Cruz, Hojancha) y los distritos peninsulares de Lepanto, Paquera y Cóbano; territorios que han mostrado una amplia relación y unidad histórica.

\section{EL SISTEMA VIAL EN LA REGIÓN CHOROTEGA}

Hasta las primeras décadas de la segunda mitad del siglo xx, predominó un sistema vial basado en la comunicación entre las haciendas, como principales polos productivos, con las poblaciones y con los puertos fluviales que comunicaban con el río Tempisque y con la zona de la península de Nicoya y del norte de Puntarenas a través de la navegación del golfo de Nicoya. Por su parte, hasta la entrada en pleno funcionamiento de la carretera Interamericana en el decenio de 1960, la principal vía terrestre hacia el centro del país fue una muy modesta e insegura carretera que se volvía intransitable durante la estación lluviosa, lo que obligaba al uso de las rutas fluviales para mantener un transporte constante $y$ seguro de personas y mercancías (Zeledón, 2009).

Para Marín y Núñez (2011a), el desarrollo del cabotaje fue resultado del interés estatal por vincular las zonas productivas aisladas con el modelo agroexportador regido desde el Valle Central. La base de este sistema fue el río Tempisque y muchos de sus afluentes como el río Bolsón y el Bebedero, junto a modestos puertos que actuaron como enlace entre las rutas terrestres y las fluviales.

De acuerdo con Marín y Núñez (2011a), las principales poblaciones y ríos en los que se sustentó el cabotaje fueron los siguientes: poblaciones costeras de Arios, Bajos Negros, Barco Quebrado, Bejuco, Cabo Blanco, Cabuya, Carrillo, Estero del Barco, Las Peladas, Lepanto, Manzanillo, Montezuma, Naranjo, Paquera, Potreros, Puerto Soley, Puerto Viejo pun, Tamarindo, Tambor, Ventanas, Sámara, Chira, Chomes, Curú, Gigante, Las Agujas, Orgores, Piedra Amarilla y San Lucas. Mientras que los principales ríos y puertos fueron: Ballena, Bebedero, Bolsón, Colorado, Corozal, Coyolar, El Charco, Isla de Hora, Jicaral, Las Letras, Pochote, Puerto Humo, Puerto Jesús, Puerto Thiel, San Pablo y Taboga. 


\section{FIGURA 1}

\section{MAPA DE LAS PRINCIPALES RUTAS FLUVIALES Y PUERTOS DE GUANACASTE}

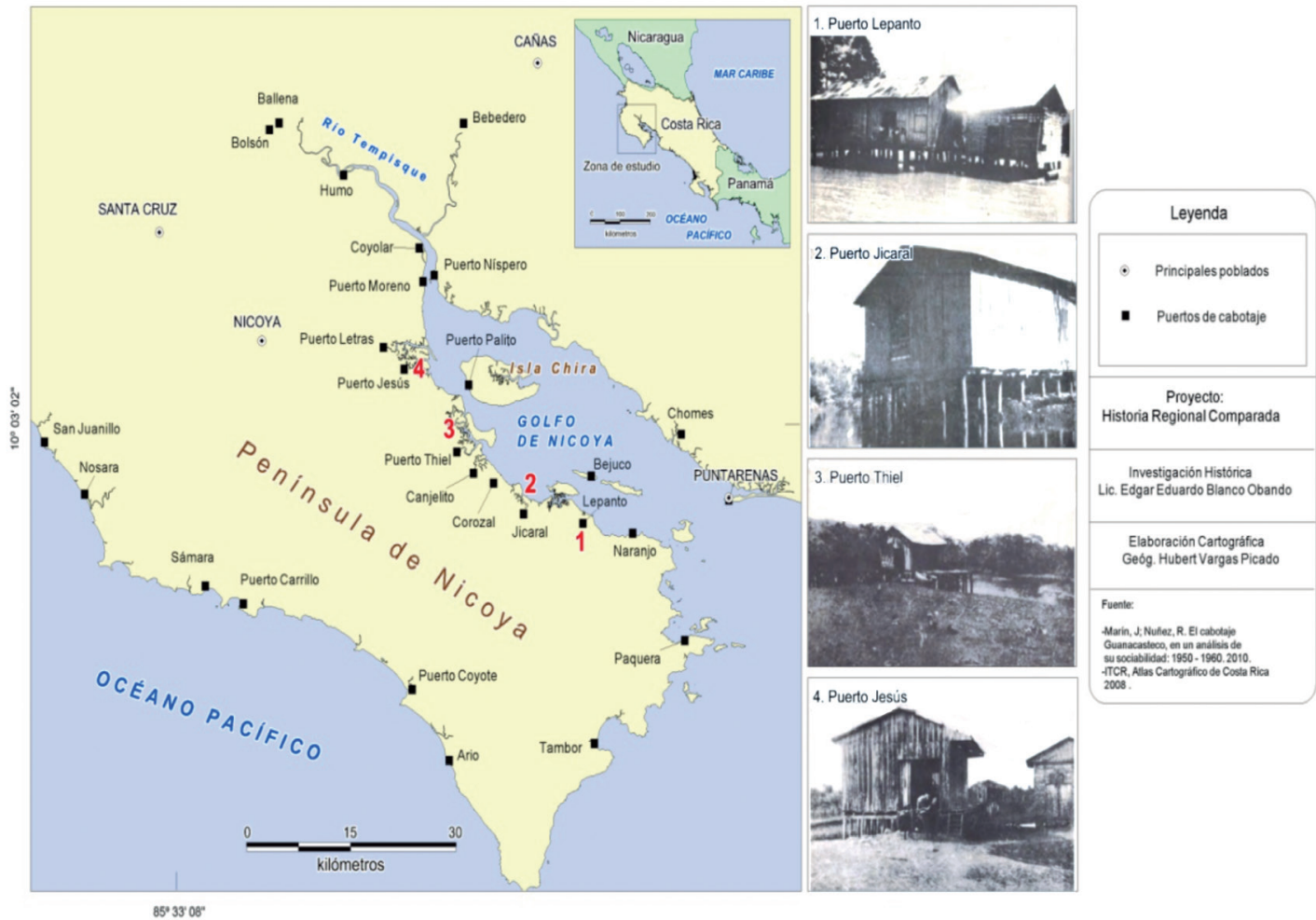

Fuente: Atlas Cartográfico de Costa Rica (p.16), Instituto Tecnológico de Costa Rica, 2008, Costa Rica: EITCR. Derechos reservados 2008 por Edgar Eduardo Blanco Obando.

Esta red fluvial aseguraba el traslado de mercancías y pasajeros tanto en invierno como en verano, asimismo permitía la comunicación con el resto del país a través de los poblados del norte de Puntarenas $y$ de su puerto conectado por ferrocarril con el Valle Central. Sin embargo, este sistema de transporte y comunicación operó con una tecnología muy rudimentaria $y$ careció de una constante inversión y mantenimiento adecuado.

La navegación dependía de la marea alta en el golfo de Nicoya que aseguraba el caudal suficiente para el zarpe de las embarcaciones en los ríos afluentes del Tempisque, mientras que los embarcaderos carecían de servicios mínimos para los viajeros, como agua potable $y$ venta de alimentos (Pizarro, 2009).
El alemán Karl Sapper, viajó en 1899 de Puerto Jesús a Puntarenas y realizó una descripción de lo modesto de los servicios y lo básico de la tecnología existente, lo cual permite comprender el estado en que operaba el sistema de cabotaje en ese entonces:

Este "Puerto" consiste en una casa, la única, situada unos pocos centenares de metros al final del estero, y el estero es un brazo de mar que penetra con sinuosidades de río, bastante profundo en el interior del terreno, $y$ en tiempo de alta marea se llena de agua que se precipita desde el mar como un torrente de considerable inclinación... "La Chepita" apenas podía avanzar con remos. Por 
fin tomó el estero una dirección exactamente hacia el este, $y$ como a la vez esta ruta era de una anchura considerable, pudimos izar nuestra vela y así navegamos cómodamente hacia la Isla de Chira, donde bajamos a tierra en la solitaria finca "La Coloradita" (Sapper citado en Meléndez, 1974, p.326).

En 1910, las características de la navegación y las condiciones de las naves fueron descritas por el estadounidense Phillip Clavert, quien en ese entonces realizó la travesía entre Bolsón y Puntarenas:

La embarcación "Castro" llegó tarde y eran las diez en vez de las nueve cuando partió. Estaba menos atestada y era más confortable que su navío hermano en el cual vinimos, pero como aquéllas, sólo tenía una cubierta; la nave podía tener unos cincuenta pies de longitud... Navegamos lentamente hacia abajo del Estero, donde el bosque llega hasta el borde del agua: ya dentro del Tempisque, atracamos y partimos de Puerto Humo, sin encallar en ningún banco de arena; tocamos Manzanillo a las 4:30 pm y llegamos a Puntarenas cerca de las ocho (Clavert citado en Meléndez, 1974, p.439).

El cabotaje vivió su mejor momento durante la primera mitad del siglo xx, cuando logró involucrar en su dinámica socio-comercial a numerosas poblaciones guanacastecas y puntarenenses, principalmente de la zona del golfo de Nicoya y del Tempisque. Sin embargo, a partir del decenio de 1950, el Estado realizó una importante inversión en tecnología e infraestructura, por lo que se introdujeron modernos medios de transporte acuáticos como "ferrys" y se mejoró significativamente la red vial terrestre, por lo que se redujo el uso del cabotaje hasta prácticamente desaparecer (Marín y Núñez, 2011b).

\section{CONSTRUCCIÓN DE LA CARRETERA INTERAMERICANA}

Esta vía posibilitó la ansiada comunicación terrestre con el Valle Central de forma directa y continua durante todo el año; su construcción se logró gracias a las acciones del gobierno de los Estados Unidos para contar con una vía única a lo largo de todo el continente que le permitiera la oportuna movilización de sus tropas y equipos militares.

Si bien, el proyecto empezó a definirse desde la década de 1940, es en el decenio de 1960 cuando la calzada es concluida, convirtiéndose rápidamente en la principal vía nacional y eje central de los caminos regionales en general (Serrano, 1976). De este modo, las poblaciones y los centros de producción en Guanacaste empezaron a conectarse con la nueva carretera, abandonando el antiguo sistema de caminos que unía a las poblaciones con las haciendas $y$ estas con los puertos fluviales (Zeledón, 2009). 


\section{FIGURA 2 \\ MAPA DEL TRAYECTO DE LA CARRETERA INTERAMERICANA \\ POR GUANACASTE Y COSTA RICA EN 1963}

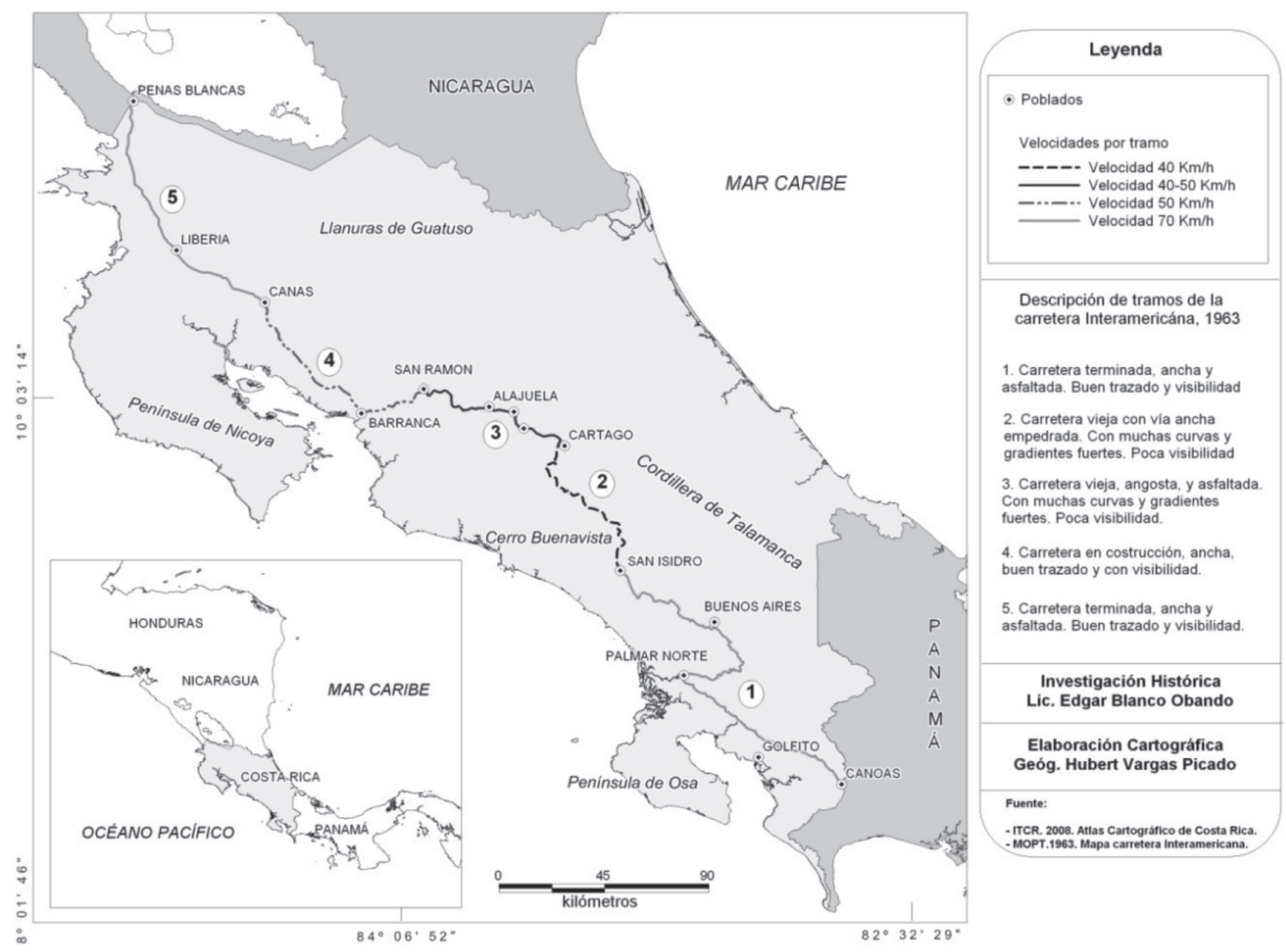

Fuente: Atlas Cartográfico de Costa Rica (p. 13), Instituto Tecnológico de Costa Rica, 2008, Costa Rica: EITcR. Derechos reservados 2008 por Edgar Eduardo Blanco Obando.

Para Zeledón (2009), la carretera Interamericana transformó el sistema vial y productivo guanacasteco, $y$ facilitó el control del espacio regional por parte del Estado, lo que terminó con el histórico aislamiento de Guanacaste con respecto al resto del país.

\section{SISTEMAS PRODUCTIVOS}

Entre la década de 1950 y la de 1980, prevaleció en la región Chorotega la agroproducción como el principal sector productivo, resultado del apoyo y de las transferencias tecnológicas realizados por parte del Estado, interesado en contar con la producción agrícola suficiente para abastecer de alimentos a la población nacional y de materias primas al sector industrial.

Se brindó apoyo al sector manufacturero $y$ a las exportaciones de productos agropecuarios mediante la modernización de las unidades productivas, junto con introducción de semillas mejoradas y la intensiva aplicación de agroquímicos, con el fin de generar recursos para financiar el crecimiento de la industria y de la economía en general (León et ál., 2014).

Como resultado de la implantación de dicho sistema, se produjo la intensificación de la producción extensiva de cultivos como el arroz y la caña de azúcar, mediante la aplicación de paquetes de modernización y mecanización agrícola, especialmente en zonas 
periféricas con ventajas topográficas, climatológicas y con eficientes vías de comunicación, debido a que permitían incrementar los terrenos y reducir los costos del transporte (Arroyo y León, 2012).

La región pasó de poseer en 1950 cerca del $10 \%$ de los productores de caña del país, a tener el 19\% en el 2000; además, en el década de 1970 se habían asentado en la región parte de los ingenios más modernos y de mayor tamaño, consolidando a Guanacaste junto con la región del Pacífico Central como las principales zonas cañeras del país. Para el caso del arroz, la apertura de las nuevas vías de comunicación fomentaron la incorporación de nuevas tierras y la aplicación de sistemas mecanizados (León, 2012).

En el proceso de desarrollo y modernización de la agricultura, fue siempre de interés estatal la construcción de sistemas de regadíos, aprovechando los caudalosos ríos de la zona. Al inicio del decenio de 1950, el entonces Ministerio de Agricultura e Industria (MAI), impulsó la construcción de obras de riego a gran escala, empleando las aguas de ríos como el Tempisque y el Corobicí:

En la finca Paso Hondo se está actualmente construyendo un canal de derivación con capacidad de 1.200 Litros por segundo que tomando las aguas del río Corobicin [Corobicí] las conduce hasta la parte más alta de la finca desde donde se comenzará un sistema de distribución para el riego de pastos $y$ de otras cosechas propias de la región; el área que se piensa abarcará la terminación del proyecto total es aproximadamente de 1.200 hectáreas (Ministerio de Agricultura e Industria, 1952, p.166).

El MAI también construyó un canal para irrigar con aguas del río Colorado, tierras en la finca La Cueva. Mientras que en la Hacienda El Pelón de la Bajura, el Ministerio apoyó la canalización de aguas del río Salto y la construcción de diques. Posteriormente, en la Hacienda El Viejo, el MAI construyó un canal de 500 metros al margen del Tempisque, para regar 200 manzanas (Ministerio de Agricultura e Industria, 1952).

Para la década de 1970 , se realizaron acciones para irrigar las tierras de la Cuenca del Tempisque con las aguas residuales del proyecto hidroeléctrico de Arenal, para lo cual se fundó en la década de 1980, el Proyecto de Riego Arenal-Tempisque administrado por el Servicio Nacional de Aguas Subterráneas, Riego y Avenamiento (senara), que se convirtió en el principal y más exitoso proyecto de riego del país (ver figura 3). El éxito del proyecto se reflejaba en el continuo incremento del área beneficiada por el riego, que a finales de la década de 1990 era 15902 ha, a mediados del decenios del 2000 alcanzó las 18400 ha y para el 2012, el riego cubrió 27000 ha. (Servicio Nacional de Aguas Subterráneas, Riego y Avenamiento, 2012). 


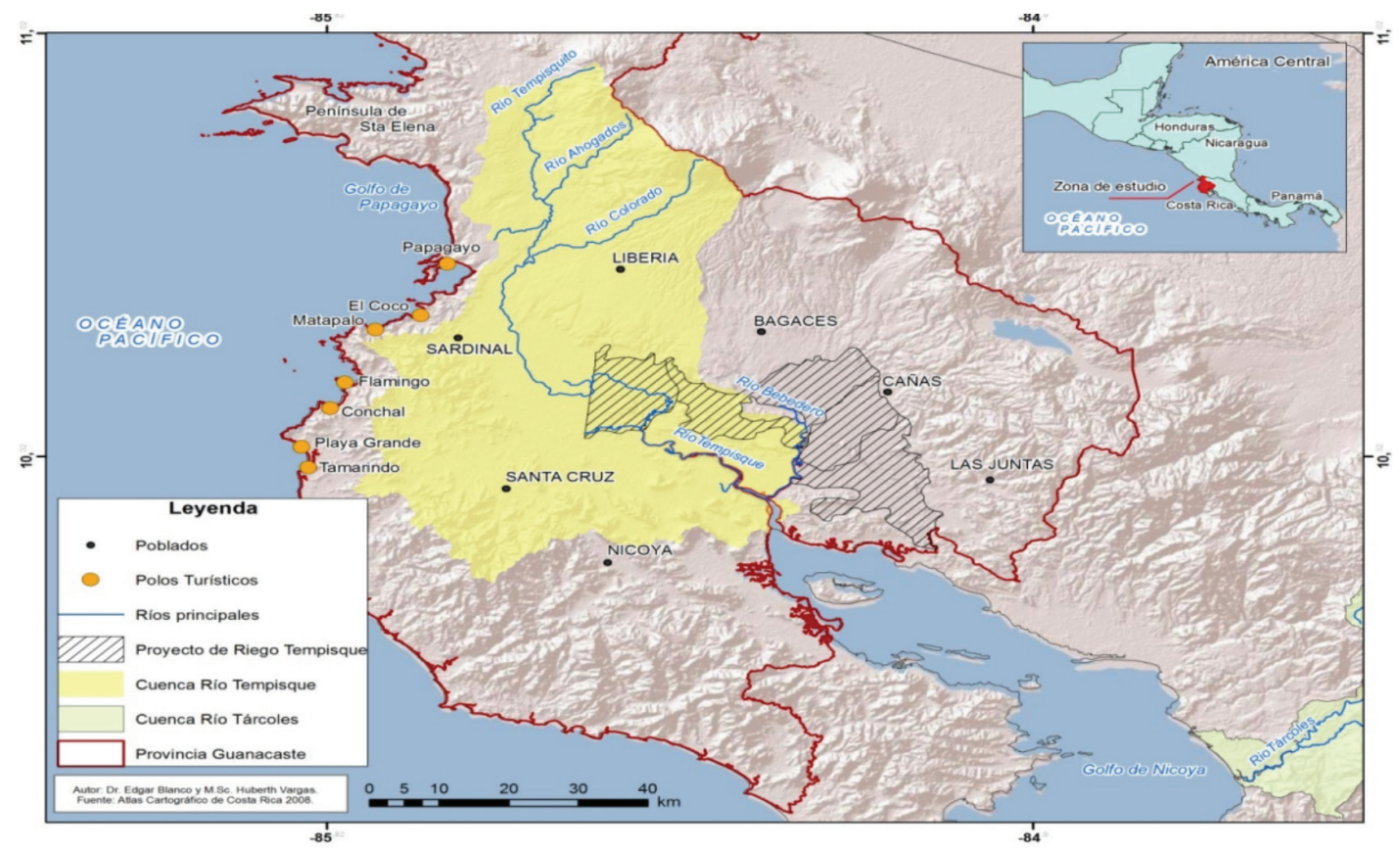

Fuente: Atlas Cartográfico de Costa Rica (p.10), Instituto Tecnológico de Costa Rica, 2008, Costa Rica: EITCR. Derechos reservados 2008 por Edgar Eduardo Blanco Obando.

A pesar del incremento en los terrenos bajo regadío público, el beneficio se concentró en los grandes terratenientes guanacastecos, los mismos que recibieron inversiones $y$ construcciones de obras en sus fincas por parte del MAI en la década de 1950, debido a que poseen la mayor cantidad de tierra dentro del proyecto de riego.

En el año 2000, el 76\% del total de las fincas dentro del distrito de riego eran menores de 10 ha, por lo que ocupaban solo el 23\% del área; mientras que las extensiones mayores de 200 ha, el 1,7\% del total de fincas con riego, cubrían el 48\% del área. De esta manera, la expansión del riego realizada por el Estado y la inversión pública, ha terminado beneficiando a una minoría de finqueros (Proyecto Estado de la Nación, 2000).

\section{TURISMO}

La actividad turística, tanto en la región Chorotega como en Costa Rica, se ha convertido en una de las principales fuentes de riqueza y empleo, esto debido al impulso estatal a partir del decenio de 1990, como resultado de la instauración del modelo de desarrollo neoliberal $y$ el traslado de las ayudas e incentivos de las actividades primarias hacia las terciarias, definidas como de mayor rentabilidad y eficiencia. De este modo, se realizó una reconversión productiva basada en el desestímulo de la producción agrícola y el fomento especialmente de las actividades turísticas.

Como resultado de dicha reconversión productiva, Guanacaste se convirtió en el territorio con mayor inversión turística del país, en detrimento del dinamismo de la producción agrícola. El principal desarrollo del turismo se ha concentrado en las zonas costeras, esencialmente en los cantones de Liberia, Carrillo y Santa Cruz bajo el modelo de sol y playa masivo (ver imagen 3).

Al inicio de la década de 1990, el total de habitaciones turísticas en Guanacaste fue de 547, 
lo que la situó como la tercera provincia con la mayor cantidad de habitaciones, por debajo de San José (618) y Puntarenas (617) (Instituto Costarricense de Turismo, 1991). Con respecto a las empresas de hospedaje en el año 2000, Guanacaste fue la tercera provincia con mayor cantidad de este tipo de negocios (298), por detrás de Puntarenas (591) y San José (334) (Instituto Costarricense de Turismo, 2001).

Sin embargo, en el 2010, la región Chorotega presentó la segunda mayor cantidad de empresas de hospedaje (25,3\%), levemente por debajo de la región Central (27\%) y superando ampliamente a la región Pacífico Central (18,3\%) (Instituto Costarricense de Turismo, 2010); junto al mayor número de habitaciones turísticas (6569), el 30,3\% del total nacional, por lo que superó ampliamente a San José (5530 - 25,5\%) y a Puntarenas (4348 - 20,1\%) (Instituto Costarricense de Turismo, 2013).

Al presentar Guanacaste la segunda mayor cantidad de empresas de hospedaje pero el mayor número de habitaciones turísticas, se demuestra que la oferta del turismo en la provincia está basada en la presencia de grandes proyectos hoteleros con cientos de habitaciones.

\section{EFECTOS SOCIALES}

Tomando en cuenta los principales indicadores del bienestar de la población guanacasteca en momentos posteriores a la consolidación de cada sistema productivo, es posible apreciar los resultados derivados a nivel social. Si bien, el fomento del desarrollo fue prácticamente el principal justificante de la implementación del cambio tecnológico para lograr la incorporación de la región al sistema económico y productivo nacional, lo cual debía producir mejoras significativas en las condiciones de vida de la población y la satisfacción de sus necesidades; sin embargo, solamente se alcanzó transformar el sistema vial y productivo, generando beneficios para sectores empresariales pero sin transformar la realidad social de la gran mayoría de la población local.

\section{DESEMPLEO}

Con respecto a la tasa de desempleo abierto, entendido como el porcentaje de población desocupada con respecto a la fuerza de trabajo, en la región Chorotega entre 1990 y el 2012, con excepción solo de 1995 y el 2013, se mantuvo en ascenso y por encima de la tasa nacional. Además, en este período la tasa de desempleo creció en un 5,4\%, superando ampliamente al crecimiento de la tasa a nivel nacional (3,9\%). Esto evidencia la consolidación del desempleo como una de las grandes problemáticas de la región Chorotega.

TABLA 1

TASA DE DESEMPLEO ABIERTO EN LA REGIÓN CHOROTEGA Y A NIVEL NACIONAL 1990-2013

\begin{tabular}{ccc}
\hline AÑO & REGIÓN CHOROTEGA & A NIVEL NACIONAL \\
\hline 1990 & 5,8 & 4,6 \\
1995 & 4,5 & 5,2 \\
2000 & 5,7 & 5,2 \\
2005 & 5,8 & 6,6 \\
2010 & 9,6 & 7,3 \\
2012 & 11,4 & 7,8 \\
2013 & 11,2 & 8,5 \\
\hline
\end{tabular}

Fuente: Adaptación de Encuesta de Hogares y Propósitos Múltiples, Instituto Nacional de Estadística y Censos (INEc), 1990, 1995, 2000, 2005, 2010, 2012, 2013, Costa Rica. 
POBREZA

El problema de la pobreza, entendida como la presencia de niveles de vida o bienestar social inaceptables debido a la imposibilidad de satisfacer las necesidades básicas, entre la década de 1980 y mediados de 1990, se mantuvo en la región muy por encima del $30 \%$, superando ampliamente al promedio nacional que apenas superó el 20\%. Por su parte, la pobreza extrema prácticamente doblegó a los porcentajes nacionales.

TABLA 2

PORCENTAJE DE HOGARES EN CONDICIÓN DE POBREZA Y POBREZA EXTREMA EN LA REGIÓN CHOROTEGA Y A NIVEL NACIONAL 1987-1996

\begin{tabular}{ccccc}
\hline \multirow{2}{*}{ AÑO } & \multicolumn{2}{c}{ REGIÓN CHOROTEGA } & \multicolumn{2}{c}{ A NIVEL NACIONAL } \\
\cline { 2 - 5 } & POBREZA & POBREZA EXTREMA & POBREZA & POBREZA EXTREMA \\
\hline 1987 & 46,2 & 21,4 & 29,0 & 9,0 \\
1995 & 35,2 & 13,6 & 20,3 & 6,2 \\
1996 & 34,6 & 13,2 & 21,5 & 6,8 \\
\hline
\end{tabular}

Fuente: $\quad$ Adaptación de Encuesta de Hogares y Propósitos Múltiples, INEc, 1990, 1995, 1996, Costa Rica.

Posteriormente, entre el 2005 y el 2013, el nivel de pobreza se mantuvo muy similar a la década de 1990, sin mostrar una reducción importante. En la región Chorotega, la pobreza convencional se redujo apenas en $0,2 \%$, mientras a nivel nacional la reducción fue del $3,1 \%$. Por su parte, la pobreza extrema aumentó en $0,8 \%$ a nivel guanacasteco, mientras que a nivel nacional lo hizo solo en un $0,4 \%$.

TABLA 3

PORCENTAJE DE POBREZA Y POBREZA EXTREMA EN LA REGIÓN CHOROTEGA Y A NIVEL NACIONAL $2005-2012$

\begin{tabular}{|c|c|c|c|c|}
\hline \multirow{2}{*}{ AÑO } & \multicolumn{2}{|c|}{ REGIÓN CHOROTEGA } & \multicolumn{2}{|c|}{ NIVEL NACIONAL } \\
\hline & POBREZA & POBREZA EXTREMA & POBREZA & POBREZA EXTREMA \\
\hline 2005 & 34,3 & 11,8 & 23,8 & 6,0 \\
\hline 2010 & 32,6 & 11,2 & 21,3 & 6,0 \\
\hline 2012 & 34,5 & 12,6 & 20,6 & 6,3 \\
\hline 2013 & 34,1 & 12,6 & 20,7 & 6,4 \\
\hline
\end{tabular}

Fuente: $\quad$ Adaptación de Encuesta de Hogares y Propósitos Múltiples, InEc, 2005, 2010, 2012, 2013, Costa Rica. 
Este comportamiento de los porcentajes mostrados evidencia que en la región Chorotega la población sufre altos niveles de pobreza de manera persistente en el tiempo.

\section{ÍNDICE DE DESARROLLO SOCIAL}

El Índice de Desarrollo Social (IDS) mide el nivel de bienestar de la población a nivel cantonal, con una escala ascendente de 0 a 100. Entre el 2001 y el 2013, los cantones guanacastecos presentaron valores entre 16,4 y 63,2 ; que corresponden a niveles bajos e intermedios de desarrollo social. Esto evidencia la permanencia en el tiempo de importantes necesidades no satisfechas entre la población de la región Chorotega.

TABLA 4

ÍNDICE DE DESARROLLO SOCIAL PARA LOS CANTONES GUANACASTECOS 2001, 2007, 2013

\begin{tabular}{llll}
\hline \multicolumn{1}{c}{ CANTÓN } & 2001 & 2007 & 2013 \\
\hline Liberia & 48,9 & 40,6 & 56,2 \\
Nicoya & 48,4 & 39,5 & 39,1 \\
Santa Cruz & 52,8 & 57,4 & 53,0 \\
Bagaces & 43,9 & 42,0 & 42,2 \\
Carrillo & 46,1 & 55,8 & 62,8 \\
Cañas & 49,9 & 37,5 & 37,2 \\
Abangares & 43,1 & 27,0 & 36,0 \\
Tilarán & 63,2 & 51,5 & 56,6 \\
Nandayure & 53,1 & 37,5 & 47,0 \\
La Cruz & 16,4 & 18,7 & 27,2 \\
Hojancha & 56,9 & 53,8 & 61,8 \\
\hline
\end{tabular}

Fuente: Adaptado de Índice de Desarrollo Social, Ministerio de Planificación Nacional y Política Económica (Mideplan), 2003, 2007, 2013, Costa Rica.

\section{DESIGUALDAD}

La condición de desigualdad se mide con el Coeficiente de Gini a través del ingreso de los hogares con valores entre cero y uno, donde cero representa la igualdad total y uno el mayor nivel de desigualdad. Entre los años 2000 y 2013, la región Chorotega mantuvo niveles de desigualdad similares al resto del país, aunque presentó un incremento del 0,066.

TABLA 5

COEFICIENTE DE GINI PARA LA REGIÓN CHOROTEGA Y A NIVEL NACIONAL 2000-2013

\begin{tabular}{ccc}
\hline AÑO & $\begin{array}{c}\text { REGIÓN } \\
\text { CHOROTEGA }\end{array}$ & $\begin{array}{c}\text { A NIVEL } \\
\text { NACIONAL }\end{array}$ \\
\hline 2000 & 0,454 & 0,413 \\
2005 & 0,396 & 0,407 \\
2010 & 0,505 & 0,535 \\
2012 & 0,513 & 0,521 \\
2013 & 0,520 & 0,508 \\
\hline
\end{tabular}

Fuente: Adaptado de Encuesta de Hogares y Propósitos Múltiples, INEC, 2013, Costa Rica.

Si bien, estos valores pueden evidenciar un nivel importante de desigualdad en la zona de estudio, es posible que sea incluso mayor, debido a que muchos sectores de la sociedad, especialmente los más altos, suelen no reportar sus verdaderos ingresos.

\section{CONCLUSIONES}

El cambio tecnológico impulsado en la región durante el período de estudio, modernizó significativamente las vías de comunicación entre la región con el Valle Central, al igual que mecanizó la agricultura y sustentó sistemas productivos modernos, dinámicos y rentables; sin embargo, con base en los indicadores de bienestar social analizados, en estos se deberían reflejar los resultados de dichos sistemas productivos; sin embargo, no se aprecia la mejora significativa de los niveles de vida de la población local, lo cual presentó la continua insatisfacción de necesidades, incluso las básicas.

Aunque se logró revertir la condición de aislamiento de la región y se modernizaron 
sus sistemas productivos de acuerdo a las principales tendencias económicas mundiales, no se logró revertir los bajos índices de bienestar social de la población. Los principales beneficiados del cambio tecnológico y la modernización de los sistemas productivos, que incorporaron a Guanacaste al mercado nacional y mundial, han sido las élites locales y vallecentralinas, junto con los sectores dominantes del comercio y la producción, que obtuvieron modernas condiciones para desarrollar sus actividades $y$ consolidar sus intereses. Por el contrario, los sectores populares fueron excluidos de los beneficios sociales de la tecnología y la modernización instaurada.

Estos resultados evidencian que aunque se han institucionalizado modelos de desarrollo modernos y dinámicos, no transformaron las condiciones sociales de la población local, la cual sufre desigualdad y exclusión de la concentración de la riqueza. El cambio tecnológico creó desarrollo económico y productivo, pero no logró la distribución equitativa de los beneficios y la mejora de las condiciones de vida de la población en la región Chorotega.

BIBLIOGRAFÍA

LIBROS

Delgado, M. M. (2004). La política rural europea en la encrucijada. Madrid, España: мapa, Serie estudios.

León, J. (2012). Historia económica de Costa Rica en el siglo Xx. (Tomo II: La economía rural). San José, Costa Rica: IICE, CIHAC, UCR.

León, J., Justo, A., Chacón, M., Peters, G., Jara, A. y Villalobos, M. (2014). Crecimiento y las políticas económicas. (Tomo I: Historia económica de Costa Rica en el siglo xx). San José, Costa Rica: EUCR.

Marín, J. y Núñez, R. (2011a). Los sistemas de cabotaje de Guanacaste, un análisis comparado de articulaciones nodales interregionales en Costa Rica. 1890-2000. En Núñez, R y Marín, J. (eds.). (Re)lecturas de Guanacaste: 1821-2010. San José, Costa Rica: Sociedad Editora Alquimia.
Marín, J. y Núñez, R. (2011b). El cabotaje guanacasteco, un análisis de sociabilidad 1950-1960. En Núñez, R y Marín, J. (eds.). (Re)lecturas de Guanacaste: 18212010. San José, Costa Rica: Sociedad Editora Alquimia.

Meléndez, C. (1974). Viajeros por Guanacaste. San José, Costa Rica: Departamento de Publicaciones, Ministerio de Cultura, Juventud y Deportes.

Ministerio de Agricultura e Industria. (1952). Memoria anual 1951. San José, Costa Rica: mai.

Rovira, J. (1987). Costa Rica en los años 80. San José, Costa Rica: Editorial Provenir.

Worster, D. (2006). Transformaciones de la tierra. Ensayos de historia ambiental. San José, Costa Rica: EunEd.

Zeledón, F. (2009). La construcción de la carretera Inter-Americana en Guanacaste, región y medios de comunicación 1943 y 1970. En Guanacaste: (Re)construcción de una región. 1850-2007. San José, Costa Rica: Librería Alma Mater.

\section{PUBLICACIONES PERIÓDICAS}

Arroyo, N. y León, J. (2012). La modernización agrícola: un proceso de largo plazo visto a través de las experiencias de la caña de azúcar y el arroz. Reflexiones, (Número especial: Jornadas Interdisciplinarias en Ciencias Sociales), 147-172.

Lepratte, L., Blanc, R., Pietroboni, R. y Hegglin, D. (Enero de 2015). Sistemas sociotécnicos e innovación. Análisis de la dinámica del sector de producción de carne aviar en la Argentina. Revista cTs, 10(28), 57-83.

Tabares, J. y Correa, S. (Mayo de 2014). Tecnología y sociedad: Una aproximación a los estudios sociales de la tecnología. Revista стs, 9(26), 129-144.

Pizarro, Y. (2009). Remontando el río, remontando el tiempo: Documentos para una historia medioambiental de la cuenca del Tempisque en la primera mitad del siglo xx. Revista Historia, (59-60), 205-223.

Viales, R. (2000b). Poblar, comunicar y buscar capitales: Tres fundamentos de la política 
agraria liberal en Costa Rica entre 1870-1930. Agronomía Costarricense, 24(1), 99-11.

TESIS

Serrano, C. (1976). Historia de la carretera Interamericana. (Tesis de Licenciatura en Historia). Universidad de Costa Rica, San José, Costa Rica.

Viales, R. (2000a). Los liberales y la colonización de las áreas de frontera no cafetaleras: El caso de la Región Atlántica (Caribe) costarricense entre 1870 y 1930. (Tesis de Doctorado en Historia). Universitat Autònoma de Barcelona, Barcelona, España.

OTROS

Costa Rica. Programa Estado de la Nación. (2000). Informe Programa: Estado de la Nación en Desarrollo Humano Sostenible (Sexto Informe, 1999). San José, Costa Rica: Programa Estado de la Nación.

Instituto Costarricense de Turismo. (1991). Memoria anual 1991. San José, Costa Rica: IcT.

Instituto Costarricense de Turismo. (2001). Memoria anual 2001. San José, Costa Rica: IcT.
Instituto Costarricense de Turismo. (2010). Memoria anual 2009. San José, Costa Rica: ICT.

Instituto Costarricense de Turismo. (2013). Anuario estadístico. Recuperado de: http//:www.visitcostarica.com/

Servicio Nacional de Aguas Subterráneas, Riego y Avenamiento. (2012). Distrito de riego ArenalTempisque. San José, Costa Rica: senara Recuperado de: http//:www.senara.go.cr/

Instituto Nacional de Estadísticas y Censos. (1990). Encuesta de Hogares y Propósitos Múltiples. Costa Rica: INEC.

Instituto Nacional de Estadísticas y Censos. (1995). Encuesta de Hogares y Propósitos Múltiples. Costa Rica: INEC.

Instituto Nacional de Estadísticas y Censos. (1996). Encuesta de Hogares y Propósitos Múltiples. Costa Rica: INEC.

Instituto Nacional de Estadísticas y Censos. (2000). Encuesta de Hogares y Propósitos Múltiples. Costa Rica: INEC.

Instituto Nacional de Estadísticas y Censos. (2005). Encuesta de Hogares y Propósitos Múltiples. Costa Rica: INEC.

Instituto Nacional de Estadísticas y Censos. (2010). Encuesta de Hogares y Propósitos Múltiples. Costa Rica: INEC.

Fecha de ingreso: 11/07/2016

Fecha de aprobación: 16/08/2016 